\title{
Maquette pédagogique pour l'enseignement des systèmes radar
}

\author{
F. VINCENT ${ }^{1}$, B. MOUTON ${ }^{2}$, C. NOUALS ${ }^{3}$ \\ Département Avionique et Systèmes, ENSICA, 1, Place Emile BLOUIN, 31056 Toulouse. \\ vincent@ensica.fr, ${ }^{2}$ mouton@ensica.fr, ${ }^{3}$ nouals@ensica.fr
}

\section{Résumé :}

De manière à faire apparaître les liens entre les différentes disciplines de base de l'enseignement EEA, le département Avionique et Systèmes de l'ENSICA (Ecole Nationale Supérieure d'Ingénieurs de Constructions Aéronautiques) propose à ses élèves en fin de $1^{\text {ère }}$ année de réaliser un convoi de maquettes de train autonomes. Par analogie avec les systèmes radar existants, les étudiants doivent développer un système ultrasonore de mesure de distance ainsi que la logique de contrôle associée.

$\mathrm{Au}$ cours de ce projet les élèves doivent faire preuve d'autonomie et d'initiative pour acquérir des connaissances sur le principe de fonctionnement des radars. Durant les 20 heures de projet encadrées, les étudiants doivent concevoir, réaliser et tester des cartes d'essai électroniques permettant au système de fonctionner. A l'issu de ce travail, ils doivent présenter leur projet devant un jury.

Mots clés : Projet pédagogique, électronique, ultrason, radar, mesure de distance.

\section{INTRODUCTION}

Les différentes matières fondamentales liées à l'électronique (automatique, traitement du signal, électromagnétisme, antennes, ...) sont souvent enseignées de manière totalement cloisonnée. Bien que beaucoup de notions sont identiques (fréquences, gains, ...), les notations et la terminologie différent souvent suivant la matière. Les étudiants ne perçoivent donc pas toujours les similitudes ou les liens entres ces différents domaines.

De manière à décloisonner ces enseignements et pour mettre en évidence les liens qu'un ingénieur doit connaître pour réaliser un système complet, l'ENSICA a développé une maquette pédagogique d'un système de contrôle de distance à destination des élèves. Cette maquette est constituée de 2 trains miniatures (cf. fig. 1). Les étudiants doivent concevoir et réaliser l'électronique embarquée de mesure et de contrôle de manière à asservir le second train sur le premier. Le train suiveur doit rester à une distance prédéfinie du premier.

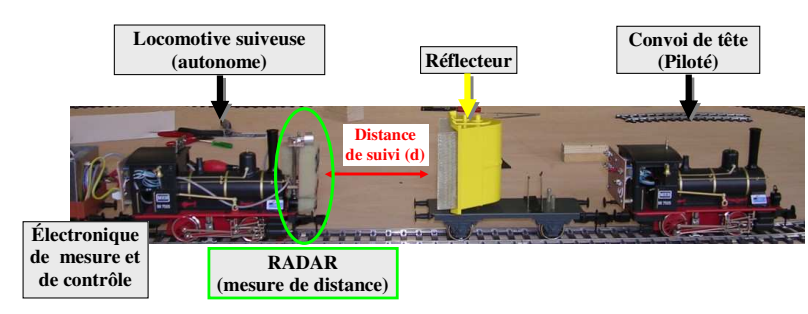

fig. 1: Photo du convoi

\section{DESCRIPTION DU PROJET}

\subsection{Matériel fourni}

Pour mener à bien leur projet, les étudiants disposent des éléments matériels suivants :

- $\quad 2$ trains miniatures à l'échelle $1 / 32$ ième de marque Marklin (cf. fig. 1)

- 2 émetteurs/récepteurs à ultrason piézoélectriques large bande de marque prowave (400 wb16)

- plaquettes d'essai électroniques labdec

- tous les composants d'électronique analogique actifs ou passifs classiques (Ampli Op., résistances, capacités, ...)

\subsection{Pré requis}

Ce projet s'adresse aux élèves ingénieurs en fin de première année. Ceux-ci ont donc les bases dans les domaines suivants :

- Electronique analogique (50 heures de formation: amplification, filtrage, oscillateurs)

- Systèmes/Traitement du signal (50 heures de formation: Laplace, Fourier, Bode, filtrage analogique et numérique)

- $\quad$ Automatique (30 heures de formation : modélisation et analyse des systèmes linéaires) 


\subsection{Travail à réaliser}

Le but fixé aux étudiants est de réaliser l'électronique embarquée permettant de mesurer la distance entre les 2 trains et de contrôler cette distance. La méthode employée pour réaliser ces 2 sous fonctions est imposée :

\subsubsection{Mesure de distance}

Le système de mesure de distance est inspiré des systèmes radar. Pour des raisons de coût et de simplicité nous avons choisi de remplacer les ondes électromagnétiques par des ondes ultrasonores. En effet, la vitesse de propagation de ces dernières étant approximativement un million de fois plus lente $\left(\mathrm{c}=343 \mathrm{~m} / \mathrm{s}\right.$ dans l'air à $20^{\circ} \mathrm{C}$ à comparer à la célérité de la lumière : $310^{8} \mathrm{~m} / \mathrm{s}$ ), nous pouvons exploiter des ondes centimétriques à des fréquences basses $\left(F_{p}=40 \mathrm{KHz}=>\lambda=8.5 \mathrm{~mm}\right)$.

De même, comme un radar mesure un retard de propagation pour estimer une distance, la précision de celle-ci sera bien meilleure pour une même incertitude sur la mesure du temps. Ainsi, l'utilisation de bandes de fréquences limitées ( $B=4 \mathrm{KHz}$, par exemple) permettent de mesurer des retards de propagation avec une résolution de l'ordre de $1 / B=0.25 \mathrm{~ms}$, et par conséquent une résolution en distance de l'ordre de $c / 2 B=4.25 \mathrm{~cm}$.

Comme dans le cas des radars à faible portée, nous avons choisi d'utiliser une onde continue et non impulsionnelle. En effet, l'émission d'une impulsion de forte puissance brouille la réception et ne permet donc pas de recevoir les échos d'objets très proches du radar. Une solution plus appropriée est de baisser la puissance de l'onde émise et d'émettre de façon continue (pour conserver une énergie émise comparable). Pour pouvoir alors distinguer l'onde reçue de l'onde émise et mesurer le retard de propagation, il faut que sa forme évolue au cours du temps. Plusieurs types de forme d'onde sont alors envisageables, nous avons choisi une forme d'onde typique et très utilisée en radar : FMCW (Frequency Modulated Continuous Wave - cf. fig 2).

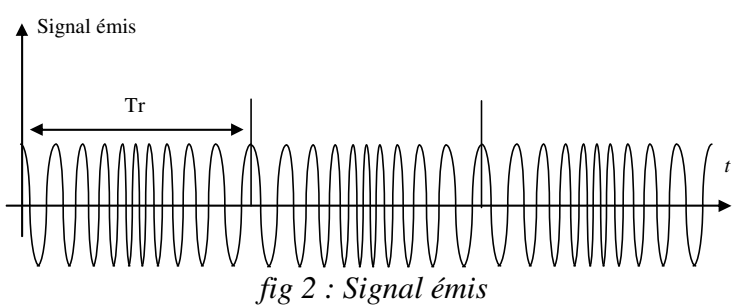

Celui-ci est modulé linéairement en fréquence comme le montre la figure 2.

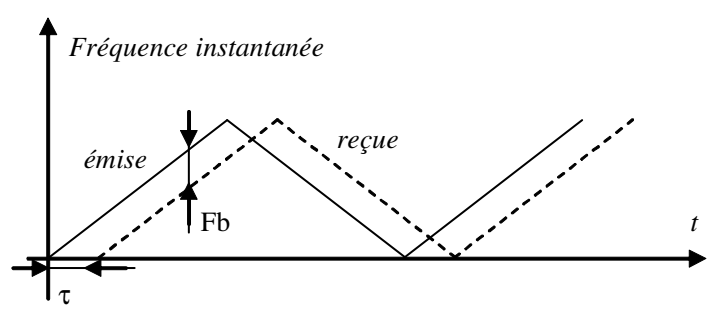

fig 3 : Fréquence instantanée de signaux émis et reçus

La figure 3 montre l'évolution fréquentielle de l'onde émise et réfléchie. Cette dernière est décalée d'un retard $\tau=2 d / c$ où $d$ représente la distance entre le radar est l'objet visé. Du fait de la linéarité de la fréquence par rapport au temps, il est alors facile de mesurer ce retard $\tau$ par l'intermédiaire de la fréquence de battement $F_{b}$ entre les 2 ondes. Celle-ci est facilement accessible en effectuant le produit du signal reçu par le signal émis et en filtrant passe-bas. En effet, le produit des 2 signaux sera constitué de 2 composantes fréquentielles. L'une évoluant en haute fréquence (autour de $2 \times F_{p}=80 \mathrm{KHz}$ ) et l'autre de fréquence fixe :

$$
F_{b}=\frac{2 B}{T_{r}} \times \frac{2 d}{c}
$$

Grâce à cette méthode, on a proportionnalité entre la distance et la fréquence. Il est alors facile de sélectionner une zone d'intérêt en distance par un filtre fréquentiel approprié. On peut par exemple ainsi limiter la distance maximale de visée ou éliminer les échos trop proches (pouvant provenir, par exemple, d'un trajet direct entre l'émetteur et le récepteur).

Pour finir, ce signal sinusoïdal dont la fréquence est proportionnelle à la distance est converti en tension continue pour être plus facilement exploitable par le système de contrôle de la distance. Cette conversion est effectuée par un convertisseur fréquence tension (LM 2917).

\subsubsection{Contrôle de distance}

Le sous-système de mesure de distance nous fournit une tension proportionnelle à la distance. Pour réguler celle-ci autour d'une valeur de consigne prédéfinie, nous allons utiliser un contrôleur de type PID (cf . fig. 4). Celui-ci devra être réalisé, comme le reste de l'électronique en analogique et pourra être simplifié en proportionnel pur dans le cadre d'une première solution.

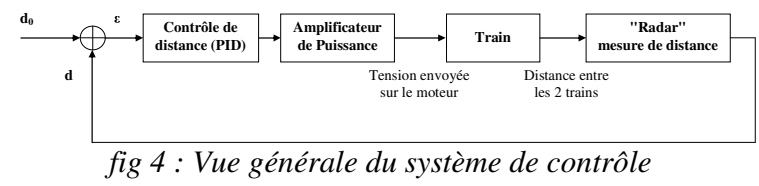


La tension de commande délivrée par le contrôleur est transmise à un amplificateur de puissance permettant d'alimenter le moteur du train suiveur.

\subsubsection{Découpage du travail à réaliser}

A partir de la description des 2 soussystèmes de mesure et de contrôle exposée précédemment, on propose aux étudiants un découpage du projet en 5 fonctions comme le montre le schéma bloc suivant (cf. fig. 5).

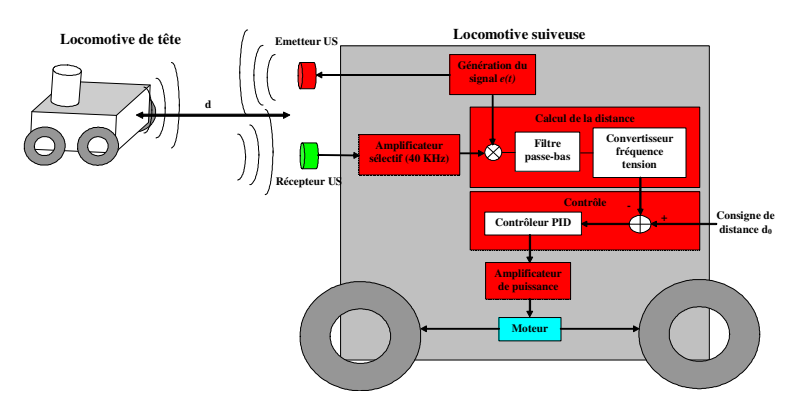

fig 5 : vue générale des différentes fonctions à réaliser

\section{EXPLOITATION PEDAGOGIQUE}

Ce projet est proposé à des groupes de 8 étudiants. Ceux-ci disposent de 20 heures réparties sur un mois pour le mener à bien. Le projet est divisé en séances de 2 heures 30 dont la moitié sont encadrées. La gestion du travail et le découpage des différentes taches du projet sont laissés au libre choix de chaque groupe. Une bonne conduite du projet est donc essentielle pour aboutir à une réalisation qui fonctionne et éviter les malentendus entre les sous-équipes chargés chacune d'un fonction particulière. On propose pour cela un découpage du projet en 7 étapes chronologiques :

1- Choix des paramètres caractéristiques de la forme d'onde à émettre. En effet, la période de répétition $\left(T_{r}\right)$, la bande du signal $(B)$ ou l'amplitude du signal émis ont des impacts directs sur la performance du système (portée du système, résolution en distance, ambiguités, ...) et doivent être choisies judicieusement. De plus, ce choix va permettre de dimensionner toutes les sous fonctions du système (largeurs de bande des filtres, périodes des oscillateurs commandant l'émission, fréquence de coupure du filtre dans le calcul de la distance, ...)

2- Définition précise des différents modules à réaliser. Cette étape doit, comme la première, être menée par la totalité du groupe de manière à définir les interfaces entres les différentes cartes électroniques (valeurs des tensions entre les modules, impédances d'entrée et de sortie, ...)
3-Synthèse des schémas électroniques permettant de réaliser chaque sous fonction. Lors de cette étape, les étudiants ont accès à une bibliothèque spécialisée en schémas électroniques (schématèque) ainsi qu'aux ressources d'Internet.

4-Réalisation des montages électroniques sur plaque d'essai.

5-Association des différents modules électroniques, réglages et test de performance.

6-Rédaction d'un rapport technique résumant le travail réalisé. Ce rapport est volontairement limité à 10 pages pour obliger les élèves à un travail de synthèse et de concision.

7-Présentation devant un jury du travail réalisé. Cette présentation est limitée à 15 minutes suivies de 15 minutes de questions pour chaque groupe.

\section{CONCLUSION}

De manière à illustrer le fonctionnement d'un système radar réel, nous avons développé une maquette de train destinée à recevoir un système embarqué de contrôle de distance. Cette maquette est exploitée au cours d'un projet de 20 heures par des étudiants travaillant en groupe de 8 . Ce projet permet de décloisonner les différentes disciplines formant l'enseignement EEA et de montrer, de façon ludique, les interactions entre ces différentes disciplines. Contrairement aux Travaux Pratiques classiques, ce projet laisse une part importante d'initiative aux étudiants et essaye d'être représentatif de la démarche que doit avoir un ingénieur.

\section{BIBLIOGRAPHIE}

1. F. Vincent, B. Mouton, C. Nouals. Outil pédagogique pour l'analyse des systèmes d'imagerie $R A D A R$. CETSIS 2005, Nancy

2. Merrill I. Skolnik. Radar Handbook. Mc GrawHill Book Compagny 1970.

3. B. Mouton, F. Vincent, C. Nouals. Réalisation d'un RADAR de poursuite. CETSIS 2003, Toulouse

4. François Le Chevalier. Principles of Radar and Sonar signal processing. Artech House Publishers 2002. 